\title{
Percutaneous Antegrade Mitral Paravalvular Leak Closure Through Porcelain Atrial Septum
}

\section{- First Percutaneous Paravalvular Leak Closure in Japan -}

Hidehiko Hara, MD; Yasushi Matsumoto, MD; Takashi Matsumoto, MD; Saibal Kar, MD;

Go Hashimoto, MD; Tadashi Araki, MD; Yoshinori Nagashima, MD; Kenji Yamazaki, MD;

Masahide Tokue, MD; Yoshinari Enomoto, MD; Fumiyuki Hayashi, MD; Yoshiyuki Yazaki, MD;

Raisuke Iijima, MD; Makoto Suzuki, MD; Kaoru Sugi, MD; Masato Nakamura, MD

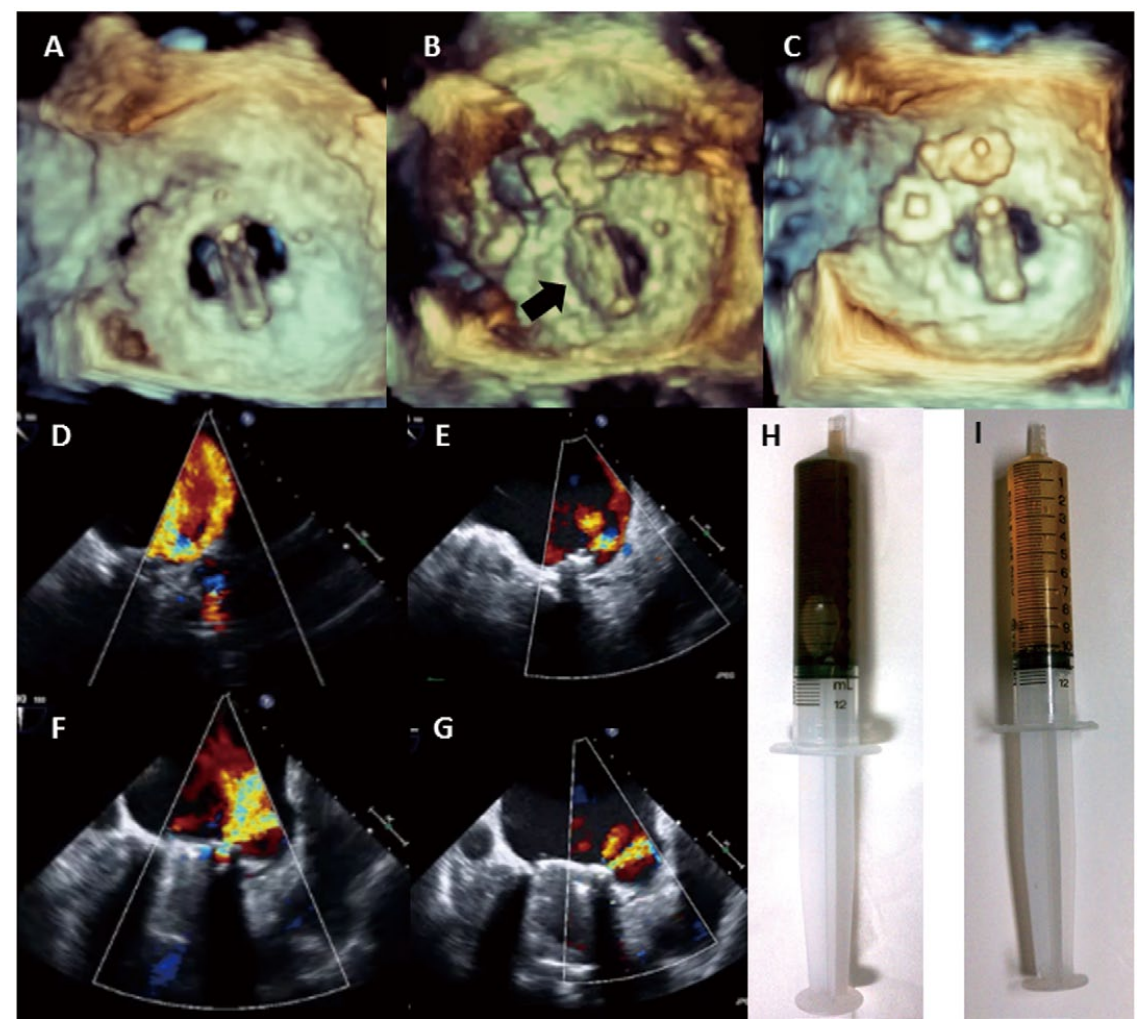

Figure 1. Before and after percutaneous paravalvular leakage (PVL) closure. (A) Live 3-D transesophageal echocardiography (3D-TEE) from the surgeon's view showing the mechanical mitral valve. The responsible site of linear leakage is not seen clearly without a color flow, located from 10 to 12 o'clock. (B) Impingement between the AMPLATZER Vascular Plug II (AVPII) and mechanical mitral valve cusp (arrow, one of the closed cusps). (C) After detachment of the three AVPII devices. (D) $0^{\circ}$ TEE obtained before closure showing significant PVL into the left atrium. (E) After PVL closure, the amount of leakage was reduced. One of the devices is seen at the edge of the prosthetic valve. (F) $90^{\circ}$ TEE showing significant PVL. (G) Following implantation of the devices, the amount of leakage obviously decreased. (H) The color of the patient's urine before closure was dark brown due to severe hemolysis, but (I) after PVL closure it changed dramatically, indicating an improvement in the hemolysis.

Received August 24, 2014; revised manuscript received December 21, 2014; accepted December 23, 2014; released online January 26, 2015 Time for primary review: 29 days

Division of Cardiovascular Medicine, Toho University Ohashi Medical Center, Tokyo (H.H., G.H., T.A., Y.N., K.Y., M.T., Y.E., F.H., Y.Y., R.I., M.S., K.S., M.N.); Department of Cardiovascular Surgery, National Hospital Organization Kanazawa Medical Center, Kanazawa (Y.M.), Japan; and Heart Institute, Cedars-Sinai Medical Center, Los Angeles, CA (T.M., S.K.), USA

Mailing address: Hidehiko Hara, MD, PhD, Division of Cardiovascular Medicine, Toho University Ohashi Medical Center, 2-17-6 Ohashi, Meguro-ku, Tokyo 153-8515, Japan. E-mail: harahide@oha.toho-u.ac.jp

ISSN-1346-9843 doi:10.1253/circj.CJ-14-0940

All rights are reserved to the Japanese Circulation Society. For permissions, please e-mail: cj@j-circ.or.jp 


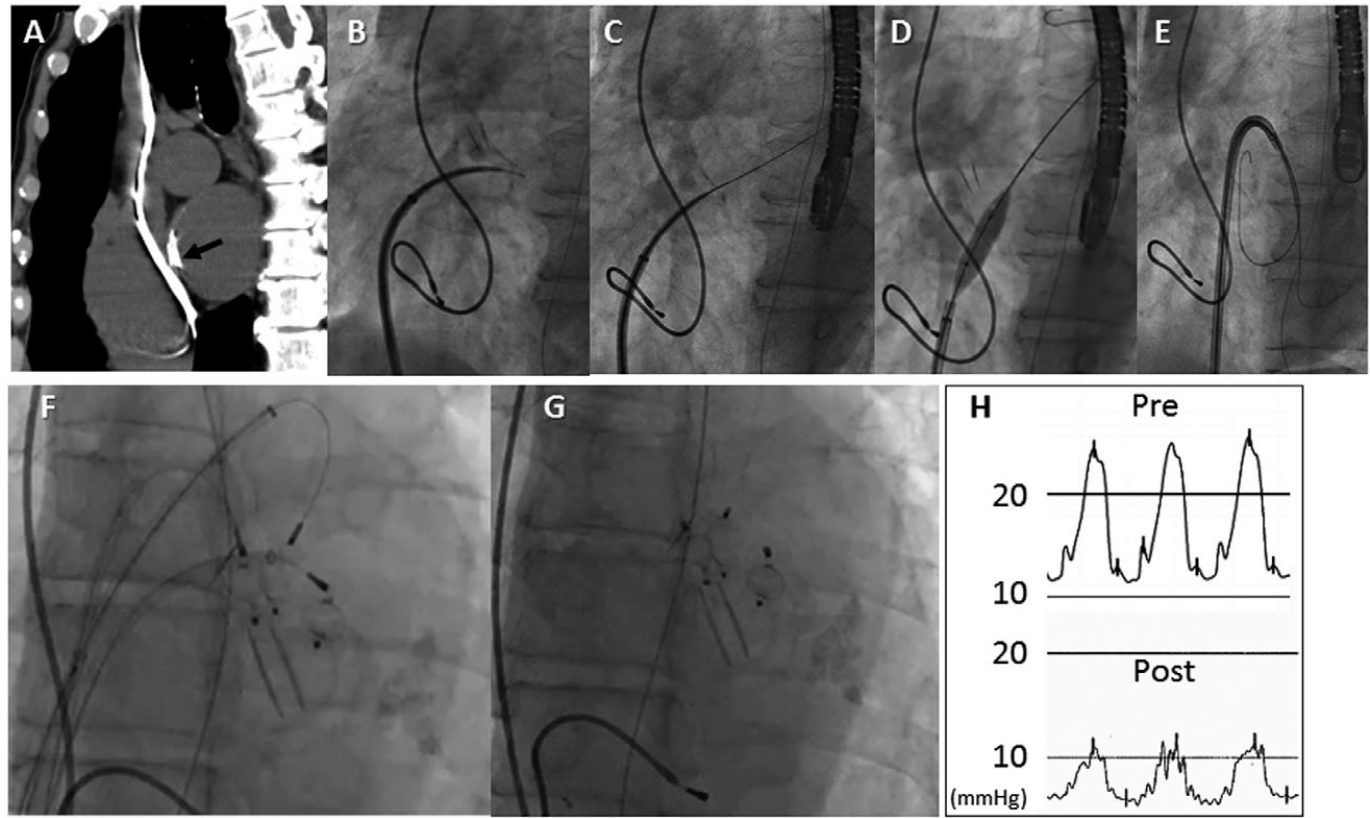

Figure 2. Hemodynamics and images obtained via computed tomography (CT) and fluoroscopy during operation. (A) Sagittal CT showing a heavily calcified atrial septum. (B) Transseptal puncture fails to penetrate the heavily calcified septum. (C) A stainless steel 0.014-inch guidewire with a hydrophilic coating was used to penetrate the heavily calcified septum to reach the left atrium. (D) Balloon septostomy to create an atrial septal defect due to the need to insert three sheaths simultaneously through the heavily calcified septum. (E) Telescoping system with a steerable introducer and guiding sheath, which enabled the insertion of a stiff 0.035 -inch guidewire into the left ventricle. (F) After deployment of the three devices, the wire cables remained connected with all devices. (G) After the release of all wire cables the valve cusp motion was not obstructed. (H) Pre- and postoperative left atrium (LA) pressure. Paravalvular leakage closure significantly reduced the LA pressure.

$\mathbf{S}$ ructural heart disease (SHD) interventions have rapidly progressed worldwide and across Japan, including the use of atrial septal defect (ASD) closure ${ }^{1}$ and transcatheter aortic valve implantation, ${ }^{2,3}$ although there are no reports of paravalvular leakage (PVL) closure in Japan to date.

PVL complicating mechanical or bioprosthetic valve replacement has been reported to occur in $2-12 \%$ of patients treated with post-mitral valve replacement and $1-5 \%$ of those undergoing post-aortic valve replacement. ${ }^{4,5}$ Surgical treatment remains the standard of care for severe PVL, but there has been great interest in the use of less invasive percutaneous approaches, which allow for the successful repair of PVL without the need for additional surgical sternotomy. ${ }^{5}$

A 68-year-old man was transferred to Toho University Ohashi Medical Center, Tokyo, to undergo percutaneous PVL closure due to severe hemolytic anemia that required treatment with $4 \mathrm{U}$ red blood cells each week, and heart failure (New York Heart Association class III). The patient underwent double valve replacement twice, in 1981 and 1989 for rheumatic valvular disease, and developed infective endocarditis 8 years after the first surgery. He also had a history of permanent pacemaker implantation to treat bradycardia.

We had several significant discussions with world experts on SHD intervention regarding imaging with transesophageal echocardiography (TEE), which included the need for live 3D-TEE, and the number of required devices. After obtaining permission from the institutional review board, given that this was the first use of this therapy in Japan and required off-label use of the device, the AMPLATZER Vascular Plug II (AVPII;

\section{St. Jude Medical [SJM], St. Paul, MN, USA).}

The patient presented with remarkable systemic jaundice (total bilirubin, $2.8 \mathrm{mg} / \mathrm{dl}$; lactate dehydrogenase, 5,794 IU) as well as mild anemia after frequent blood transfusions (hemoglobin, $9.7 \mathrm{~g} / \mathrm{dl}$; ferritin, $2,910 \mathrm{ng} / \mathrm{ml}$; haptoglobin, $<10 \mathrm{mg} / \mathrm{dl}$ ). He was receiving $3.75 \mathrm{mg}$ warfarin, $200 \mathrm{mg}$ diltiazem, $100 \mathrm{mg}$ allopurinol and $10 \mathrm{mg}$ pravastatin. We added i.v. antibiotics and heparin to regimen prior to the current procedure.

The color of the patient's urine before treatment was dark brown (Figure 1H), indicating severe hemolysis. The indications for percutaneous PVL closure in this case included both severe hemolytic anemia and heart failure with a high surgical risk (The Society of Thoracic Surgeons score, $11.1 \%$; Japan score, $36.1 \%)$.

We subsequently administered general anesthesia, and started the procedure with live 3D-TEE using CX50 xMATRIX Ultrasound system and cxX7-2t transducer (Philips Healthcare, Andover, MA, USA) and a biplane cineangiogram. We also used QLAB 3D Quantification for 3-D analysis and QLAB Mitral Valve Quantification especially for creating 3-D mitral valve (Philips Healthcare; Figures 1A-C). Puncture sites were created in the bilateral femoral vein. One of the most challenging steps was to perform transseptal puncture (TSP), given that the atrial septum was heavily calcified (Figure 2A), resembling pottery heart. ${ }^{6} \mathrm{We}$ used Agilis 8.5-Fr sheath (SJM) and attempted to place the TSP needle several times, changing the configuration of the needle each time, but were not successful (Figure 2B). Therefore, we used Swartz SL0 sheath (SJM) and Astato XS 9-12 (Asahi Intecc, Nagoya, Japan), a 
stainless steel wire usually used to penetrate sites of chronic total occlusion (CTO) in peripheral arteries (Figure 2C). Finally, we were successful in penetrating the heavily calcified atrial septum and were able to insert the dilator of the sheath into the left atrium. We then changed the 0.035-inch Amplatz super stiff wire (Boston Scientific, Marlborough, MA, USA) and dilated the septum using a $10 \times 20-\mathrm{mm}$ diameter Mustang balloon (Boston Scientific) to create the ASD (Figure 2D). Atrial septostomy effectively allowed insertion of three Destination sheaths (Terumo, Tokyo, Japan) simultaneously to deploy the devices. We subsequently changed the SLO to a telescoping system using an Agilis and Destination and manipulated the Agilis and Destination using a hydrophilic 0.035-inch wire toward the left ventricle (LV) through the linear PVL. After the wire was inserted into the LV through the PVL, the first Destination was placed within the LV, and after removing the dilator, we simultaneously inserted two long Amplatz super stiff wires curved by hand into a ram's horn shape and left inside the LV to support the subsequently inserted 6-Fr Destination into the LV at the same time (Figure 2E). We repeated this strategy again and succeeded in placing three stiff wires and three sheaths into the LV simultaneously.

The defect was linear and appeared to be located next to the left atrial appendage in the 10-12 o' clock direction within the surgeon's view (Figure 1A), with a length of $13 \mathrm{~mm}$.

We planned to implant 2-3 devices based on our discussions, but, when we actually deployed the three devices, they had to be implanted too close together in the 11-12 o'clock direction. Therefore, we attempted to recapture the last device to change its location. Unfortunately, it was impossible to drive the catheter and wire cable to allow us to change the original position of the device, and it dropped backward into the left atrium when we pulled on it. The amount of leakage from the direction of the left atrial appendage continued to be significant, which prevented us from finishing the procedure. We then inserted a steerable introducer again through the created ASD into the left atrium and selected a 5-Fr JR4 catheter to place the wire in the ideal position. We also attempted to place the wire in the $9-10$ o'clock direction to minimize the amount of leakage under live 3D-TEE navigation and fluoroscopy. After changing the JR4 to a 5-Fr LIMA catheter due to acute angulation, the wire penetrated the target lesion, and we successfully manipulated the sheath into the LV. After inserting two stiff wires, we deployed the third and fourth AVPII devices in the 9-10 o'clock direction. In total, we deployed four AVPII devices and succeeded in almost completely stopping the leakage, but, when we carefully inspected the TEE, two of four instruments were found to be stuck on the LV side of the valve cusp, which prevented the motion of one of the cusps (Figure 1B). Hence, it was necessary to remove one of the devices, although the cusp motion did not improve. The cable of the device implanted in the last instrument was pulled and pushed to change its location, and the cusp finally began to move again when the device was pushed into the LV far enough to separate from the cusp inside the LV (Figure 1C).

We subsequently detached all cables (Figures 2F,G). Although a small amount of leakage remained, it was decreased compared to that observed previously (Figures 1D-G). The mean left atrial pressure improved from 17 to $9 \mathrm{mmHg}$, and the $\mathrm{V}$ wave decreased from 25 to $13 \mathrm{mmHg}$ (Figure $2 \mathrm{H}$ ).

The day after the procedure, the patient seemed to recover from the severe hemolysis following a significant improvement in the color of his urine (Figures 1H,I). The level of lactate dehydrogenase improved from 7,136 to 2,116 IU/L at discharge. He was then transferred back to the original institution and subsequently discharged on foot.

Although we encountered difficulties due to the porcelain atrial septum, which required the use of a CTO guidewire for TSP, and the need for atrial septostomy, 2D- and 3D-TEE guidance helped us to manage these rare issues during PVL closure. In addition, TEE was key in allowing us to successfully perform a technique that has been described only rarely in the previous literature when facing impingement between the plug and mechanical valve cusp.

We herein describe successful percutaneous PVL closure through a porcelain atrial septum, the first such case reported in Japan. We believe that the advances in imaging have made it possible to more easily perform this complicated procedure.

\section{References}

1. Hara H, Kawamura A, Shirai S, Tada N, Nakamura T, Hosokawa S, et al. Progress of percutaneous atrial septal defect closure: Study from the multicenter registry of Japanese Association of Cardiovascular Intervention and Therapeutics institutions. J JCS Cardiologists 2013; 21: $256-261$.

2. Hara H, Schwartz RS. Transcatheter aortic valve implantation in high-risk patients with severe aortic stenosis. Circ J 2010; 74: 1513 1517.

3. Sawa Y, Saito S, Kobayashi J, Niinami H, Kuratani T, Maeda K, et al; MDT-2111 Japan Investigators. First clinical trial of a selfexpandable transcatheter heart valve in Japan in patients with symptomatic severe aortic stenosis. Circ J 2014; 78: 1083-1090.

4. Rihal CS, Sorajja P, Booker JD, Hagler DJ, Cabalka AK. Principles of percutaneous paravalvular leak closure. JACC Cardiovasc Interv 2012; 5: $121-130$

5. Krishnaswamy A, Kapadia SR, Tuzcu EM. Percutaneous paravalvular leak closure: Imaging, techniques and outcomes. Circ J 2013; 77: 19-27.

6. Hara H, Kimura K, Mackey-Bojack S, Schwartz RS. Pottery heart: A case of porcelain heart. Cardiovasc Revasc Med 2007; 8: 80-81. 